\title{
Heat Trace Asymptotics on Noncommutative Spaces ${ }^{\star}$
}

\author{
Dmitri V. VASSILEVICH \\ $\dagger$ Instituto de Física, Universidade de São Paulo, \\ Caixa Postal 66318 CEP 05315-970, São Paulo, S.P., Brazil \\ E-mail: dmitry@dfn.if.usp.br \\ $\ddagger$ V.A. Fock Institute of Physics, St. Petersburg University, Russia
}

Received August 30, 2007; Published online September 25, 2007

Original article is available at http://www.emis.de/journals/SIGMA/2007/093/

\begin{abstract}
This is a mini-review of the heat kernel expansion for generalized Laplacians on various noncommutative spaces. Applications to the spectral action principle, renormalization of noncommutative theories and anomalies are also considered.
\end{abstract}

Key words: heat trace asymptotics; noncommutative field theory

2000 Mathematics Subject Classification: 81T75; 58B34

Dedicated to the memory of Tom Branson

\section{Introduction}

Let us start with a brief description of the standard set-up for studying the heat trace asymptotics on a commutative space. Further details can be found in the monograph by Gilkey [25]. Let $M$ be a compact Riemannian manifold of dimension $m$, and let $D$ be an operator of Laplace type acting on the space of smooth sections of a vector bundle $V$ over $M$. In a local basis any Laplace type operator can be represented as

$$
D=-\left(g^{\mu \nu} \partial_{\mu} \partial_{\nu}+a^{\sigma} \partial_{\sigma}+b\right),
$$

where $g^{\mu \nu}$ is the inverse metric on $M$, and $a^{\sigma}$ and $b$ are matrix-valued functions. There is a unique connection $\nabla=\partial+\omega$ on $V$ and a unique endomorphism $E$ of $V$ such that

$$
D=-\left(g^{\mu \nu} \nabla_{\mu} \nabla_{\nu}+E\right) .
$$

For a positive $t$ the heat operator $e^{-t D}$ associated to $D$ exists and is trace class. Let $f$ be a smooth smearing function on $M$. As $t \rightarrow+0$ there is a full asymptotic expansion (called the heat kernel expansion in physics literature)

$$
\operatorname{Tr}\left(f e^{-t D}\right) \simeq \sum_{n=0} t^{(n-m) / 2} a_{n}(f, D) .
$$

The heat kernel coefficients $a_{n}(f, D)$ are locally computable, i.e. they can be expressed through integrals of local invariants constructed from the symbol of $D$. If $M$ has no boundary ${ }^{1}, \partial M=\varnothing$,

\footnotetext{
*This paper is a contribution to the Proceedings of the 2007 Midwest Geometry Conference in honor of Thomas P. Branson. The full collection is available at http://www.emis.de/journals/SIGMA/MGC2007.html

${ }^{1}$ The work by Tom Branson and Peter Gilkey [5] devoted to the heat trace asymptotics on manifolds with boundary is perhaps the best known to physicists paper by Tom. This paper was, in a sense, the starting point of my collaboration with Tom. In [42] I corrected a minor error of [5] made in the $a_{4}$ coefficient for mixed boundary conditions. The reaction of Tom and Peter was very friendly, and they invited me to join them and calculate the next coefficient $a_{5}$. We performed this calculation [6], though with some restrictions on the boundary conditions (pure Dirichlet or Neumann), and on the manifold (domain in flat space or totally geodesic boundaries). The restrictions on the manifold were removed by Klaus Kirsten [31]. Later, when Klaus joined the collaboration, it became possible to do $a_{5}$ for generic mixed boundary conditions [7].
} 
the odd-numbered coefficients vanish, $a_{2 k-1}(f, D)=0$, while leading even-numbered coefficients read

$$
\begin{aligned}
a_{0}(f, D)= & (4 \pi)^{-m / 2} \int_{M} d^{m} x \sqrt{g} \operatorname{tr}_{V}(f), \\
a_{2}(f, D)= & (4 \pi)^{-m / 2} \frac{1}{6} \int_{M} d^{m} x \sqrt{g} \operatorname{tr}_{V}(f(6 E+R)), \\
a_{4}(f, D)= & (4 \pi)^{-m / 2} \frac{1}{360} \int_{M} d^{m} x \sqrt{g} \operatorname{tr}_{V}\left(f \left(60 \nabla^{2} E+60 R E+180 E^{2}\right.\right. \\
& \left.\left.+12 \nabla^{2} R+5 R^{2}-2 R_{\mu \nu} R^{\mu \nu}+2 R_{\mu \nu \rho \sigma} R^{\mu \nu \rho \sigma}+30 \Omega_{\mu \nu} \Omega^{\mu \nu}\right)\right) .
\end{aligned}
$$

Here $\Omega_{\mu \nu}=\partial_{\mu} \omega_{\nu}-\partial_{\nu} \omega_{\nu}+\omega_{\mu} \omega_{\nu}-\omega_{\nu} \omega_{\nu}$ is the bundle curvature, $R_{\mu \nu \rho \sigma}, R_{\mu \nu}$ and $R$ are the Riemann curvature tensor, the Ricci tensor and the curvature scalar, respectively. Later on we shall use these formulae to compare them to the noncommutative (NC) case.

The coefficients $a_{n}$ carry a lot of important mathematical information about the bundle $V$ and the operator $D$. The Index is perhaps the most notable example. The significance of these coefficients to physics is even greater. The coefficients $a_{n}$ define the one-loop divergences in quantum field theory, the quantum anomalies, some expansions of the effective action, etc. For more information the interested reader may consult [24, 25, 32, 43, 45].

The aim of this paper is to show how and to which extent this scheme can be extended to noncommutative spaces. To describe a noncommutative deformation of a given manifold $M$ one takes the algebra $\mathcal{A}$ of smooth functions on $M$ and deforms it to an algebra $\mathcal{A}_{\Theta}$, which will be assumed associative but not commutative. Usually, such a deformation can be realized again on smooth functions on $M$, but with the point-wise product replaced by a noncommutative product.

This paper is organized as follows. In the next section we briefly discuss the spectral action principle which is one of the most important application of heat kernel expansion on NC manifolds. Then we turn to the Moyal type NC products and describe corresponding heat trace asymptotics in some detail. Other NC spaces are considered in Section 4. Section 5 contains a couple of examples of the use of the heat kernel in renormalization and calculations of the anomalies. Concluding remarks are given in the last section.

\section{Application: spectral action principle}

In noncommutative geometry [12] there is one specific application of the heat kernel expansion which is related to the spectral action principle [8]. This action is constructed from the spectral triple $(\mathcal{A}, \mathcal{H}, \not D)$ consisting of an algebra $\mathcal{A}$ acting on a Hilbert space $\mathcal{H}$ and of a Dirac operator $\not D$ which describes fluctuating fields (e.g., $\not D$ may depend on a gauge connection). The spectral action for these fluctuating fields is then defined as $\operatorname{Tr}\left(\Phi\left(\not D^{2} / \Lambda^{2}\right)\right)$, where $\Phi$ is a positive even function, $\Lambda$ is a mass scale parameter. For large $\Lambda$ the spectral action can be expanded as

$$
\operatorname{Tr}\left(\Phi\left(\not D^{2} / \Lambda^{2}\right)\right) \simeq \sum_{k=0} \Lambda^{m-k} \Phi_{k} a_{k}\left(1, \not D^{2}\right)
$$

where $\Phi_{k}$ are defined through the Laplace transform of $\Phi$ (see [35]). The Dirac operator squared is a Laplace type operator. Therefore, the heat kernel expansion allows to evaluate the $\Lambda$ expansion of the spectral action. Note, that direct calculations of the spectral action without use of the heat kernel are very cumbersome (though possible, cf. [17]). Some examples of spectral actions will be briefly discussed below. 


\section{Moyal spaces}

The most popular example of a noncommutative associative product is the Moyal product (also known as the Groenewold-Moyal or Moyal-Weyl product) which can be written as

$$
(f \star g)(x)=\left.\exp \left(\frac{i}{2} \Theta^{\mu \nu} \partial_{\mu}^{x} \partial_{\nu}^{y}\right) f(x) g(y)\right|_{y=x},
$$

where $\Theta$ is a constant skew-symmetric matrix. As it stands, the product (6) is valid for smooth functions, but by going to the Fourier transforms one can extend this definition to the case when at least one of the functions is less smooth. The definition (6) assumes existence of a global coordinate system on $M$ at least in the $\mathrm{NC}$ directions. The properties of the heat kernel expansion depend crucially on the compactness (or non-compactness) of these NC directions.

From the definition (6) it follows that

$$
e^{i k x} \star f(x)=f(x+\Theta k / 2) e^{i k x}, \quad f(x) \star e^{i k x}=f(x-\Theta k / 2) e^{i k x} .
$$

A suitable generalization of the Laplace operator (1) for Moyal spaces which covers most of the applications reads

$$
\begin{aligned}
& D=-\left(g^{\mu \nu} \nabla_{\mu} \nabla_{\nu}+E\right), \quad \nabla_{\mu}=\partial_{\mu}+\omega_{\mu}, \\
& \omega_{\mu}=L\left(\lambda_{\mu}\right)-R\left(\rho_{\mu}\right), \\
& E=L\left(l_{1}\right)+R\left(r_{1}\right)+L\left(l_{2}\right) R\left(r_{2}\right),
\end{aligned}
$$

where $L$ and $R$ are left and right Moyal multiplications, $f_{1} \star f_{2}=L\left(f_{1}\right) f_{2}=R\left(f_{2}\right) f_{1}$. External fields $l_{1,2}, r_{1,2}, \lambda_{\mu}$ and $\rho_{\mu}$ are supposed to be smooth and rapidly decaying at the infinity (if $M$ has non-compact dimensions). In this section (except for one Subsection 3.4) we assume that the metric $g^{\mu \nu}$ is constant. One can add, if needed, more terms consisting of the multiplication operators to the right hand sides of (8) and (9).

The smearing function in (2) should also be replaced by a product of Moyal multiplications, so that we shall consider asymptotic properties of the expression

$$
K(l, r, D)=\operatorname{Tr}\left(L(l) R(r) e^{-t D}\right) .
$$

Our general strategy of calculation of the heat kernel expansion is to isolate the free Laplacian $\Delta=-g^{\mu \nu} \partial_{\mu} \partial_{\nu}$ in $D$, keep $e^{-t \Delta}$ and expand the rest of the exponential. When applying this procedure, one has to evaluate the asymptotic expansion of the expressions like

$$
T(\bar{l}, \bar{r}):=\operatorname{Tr}\left(L(\bar{l}) R(\bar{r}) e^{-t \Delta}\right),
$$

where $\bar{l}$ and $\bar{r}$ are some polynomials of the smearing functions, background connections $\lambda$ and $\rho$, the potential $E$ and their derivatives. Note, that the corresponding expressions with only left or only right Moyal multiplications, $\operatorname{Tr}\left(R(\bar{r}) e^{-t \Delta}\right)$ and $\operatorname{Tr}\left(L(\bar{l}) e^{-t \Delta}\right)$ do not depend on $\Theta$, and, therefore, the heat trace asymptotics for the operators with just one type of the multiplications look as in the commutative case (see (3)-(5) above) up to replacing ordinary products by star products $[44,21]$.

In generic case, i.e. when both $\bar{l}$ and $\bar{r}$ are present, in order to evaluate the trace in (10) one has to sandwich the expression under the trace between two plane waves $e^{i k x}$ and integrate (or sum up, in the compact case) over the momenta $k$. It is convenient to make the Fourier transform of $\bar{l}$ and $\bar{r}$. After performing some obvious integrations one gets (see [47] for details)

$$
T(\bar{l}, \bar{r}) \sim \oint d^{m} k £ d^{m} q e^{-t k^{2}} \bar{l}_{-q} \bar{r}_{q} e^{-i k \wedge q},
$$

where the symbol \& denotes an integral (resp., a sum) over the momenta for noncompact (resp., for compact) dimensions. $\bar{l}_{q}$ and $\bar{r}_{q}$ denote Fourier components of $\bar{l}$ and $\bar{r}$, and $k \wedge q:=k_{\mu} \Theta^{\mu \nu} k_{\nu}$. We dropped an irrelevant overall factor in (11). Now we are ready to discuss the small $t$ asymptotic expansion of $T(\bar{l}, \bar{r})$. The result depends crucially on compactness of the manifold $M$. 


\subsection{Moyal plane}

Let us consider the Moyal plane $\mathbb{R}_{\Theta}^{m}$. The integral over $k$ in (11) can be easily performed yielding [47]

$$
T(\bar{l}, \bar{r})=(4 \pi t)^{-m / 2} \int d^{m} q \bar{l}_{-q} \bar{r}_{q} \exp \left(-\frac{1}{4 t} \Theta^{\mu \rho} \Theta_{\rho}^{\nu} q_{\mu} q_{\nu}\right) .
$$

If $\Theta \equiv 0$ one can return in the equation above to the coordinate space. The resulting expression is just a smeared heat kernel for the free Laplacian on the commutative plane. Therefore, we simply confirm a correct value for $a_{0}(l r, \Delta)$, see (3).

Let us assume that $\Theta$ is non-degenerate (which implies that $m$ is even). Then the exponent in (12) provides a suppression of contributions from large $q$. Therefore, to evaluate the behavior of (12) at small $t$ one has to expand $\bar{l}_{-q} \bar{r}_{q}$ in Taylor series around $q=0$ and then integrate over $q$. One obtains the following expression [47]

$$
T(\bar{l}, \bar{r})=(\operatorname{det} \Theta)^{-1}\left(\bar{l}_{0} \bar{r}_{0}+\mathcal{O}(t)\right) .
$$

This expression is nothing else than the heat kernel expansion for the free Laplacian $\Delta$. Comparing (13) to the the expansion (2) in the commutative case, one observes some important differences. The expansion (13) (i) does not contain negative powers of $t$, (ii) is non-local, and (iii) is divergent in the limit $\Theta \rightarrow 0$.

For applications it is important to know the asymptotic expansion on the unsmeared heat trace $\operatorname{Tr}\left(e^{-t D}\right)$. Such an expansion cannot be obtained by simply taking $l=r=1$ in the expressions above since constant functions on $\mathbb{R}^{m}$ are not Schwartz class. Moreover, $\operatorname{Tr}\left(e^{-t D}\right)$ contains a trivial volume divergence which must be regularized away by subtracting the (unsmeared) heat trace of $\Delta$. The methods described above are still applicable, and after some calculations one obtains [47]

$$
\begin{aligned}
& \operatorname{Tr}\left(e^{-t D}-e^{-t \Delta}\right) \simeq \sum_{n=2} t^{(n-m) / 2} a_{n}^{\mathrm{sub}}(D), \\
& a_{n}^{\mathrm{sub}}(D)=a_{n}^{L}(D)+a_{n}^{R}(D)+a_{n}^{\operatorname{mix}}(D),
\end{aligned}
$$

where the coefficients $a_{n}^{L}$ (respectively, $a_{n}^{R}$ ) depend on left (respectively, on right) fields only. In terms of the fields appearing in (8), (9) two leading coefficients read

$$
\begin{aligned}
& a_{2}^{L}(D)=(4 \pi)^{-m / 2} \int d^{m} x l_{1}(x), \\
& a_{4}^{L}(D)=(4 \pi)^{-m / 2} \frac{1}{12} \int d^{m} x\left(6 l_{1} \star l_{1}+\Omega_{\mu \nu}^{L} \star \Omega^{L \mu \nu}\right),
\end{aligned}
$$

where $\Omega_{\mu \nu}^{L}=\partial_{\mu} \lambda_{\nu}-\partial_{\nu} \lambda_{\mu}+\lambda_{\mu} \star \lambda_{\nu}-\lambda_{\nu} \star \lambda_{\nu}$. The coefficients $a_{2}^{R}$ and $a_{4}^{R}$ are obtained from (14) and (15) by replacing $\left(l_{1}, \lambda, \Omega^{L}\right)$ with $\left(r_{1}, \rho, \Omega^{R}\right)$. (Note, that $a_{0}^{L, R}=0$ due to the subtraction of the free heat kernel). The coefficients $a^{L}$ and $a^{R}$ are called planar because of their similarity to planar diagrams of quantum field theory.

Mixed coefficients $a_{n}^{\text {mix }}$ vanish for $n \leq m$. The first non-zero coefficient is

$$
a_{m+2}^{\operatorname{mix}}(D)=\frac{(\operatorname{det} \Theta)^{-1}}{(2 \pi)^{m}}\left(\int d^{m} x l_{2}(x) \int d^{m} y r_{2}(y)+2 \int d^{m} x \lambda_{\mu}(x) \int d^{m} y \rho^{\mu}(y)\right) .
$$

This coefficient is non-local and divergent in the limit $\Theta \rightarrow 0$, as expected.

If $\Theta$ is degenerate, there can be mixed non-local heat kernel coefficients $a_{n}^{\text {mix }}$ for $n \leq m$. For the reasons explained below, this can lead to problems with the renormalizability [19].

Heat kernel expansion for non-minimal operators, such as appear in NC gauge theories, was constructed in [39]. 


\subsection{NC torus}

Let us now turn to the noncommutative torus $\mathbb{T}_{\Theta}^{m}$. This space was first constructed by Connes [11]. The heat kernel expansion on $\mathbb{T}_{\Theta}^{m}$ was analyzed in [23]. Below we give a brief overview of the results. On $\mathbb{T}_{\Theta}^{m}$ it is not that essential whether $\Theta$ is degenerate or not, but instead some number theory aspects play an important role.

One can bring $T(\bar{l}, \bar{r})$ to the form

$$
T(\bar{l}, \bar{r})=\sqrt{g}(4 \pi t)^{-m / 2} \sum_{q \in \mathbb{Z}^{m}} \sum_{k \in \mathbb{Z}^{m}} \bar{l}_{-q} \bar{r}_{q} e^{-\frac{|\theta q-2 \pi k|^{2}}{4 t}},
$$

where $g$ is determinant of a (constant) metric on $\mathbb{T}^{m}$. Each term under the sum in (16) is exponentially small at $t \rightarrow 0$ unless $\theta q-2 \pi k=0$ for some $q$ and $k$. However, this "smallness" can be overcompensated by an infinite number of terms in the sum. To understand better what is going on, let us consider the case when $\Theta$ has a block-diagonal form

$$
\Theta=\bigoplus_{\theta_{i} \in 2 \pi \mathbb{Q}} \theta_{i}\left(\begin{array}{cc}
0 & 1 \\
-1 & 0
\end{array}\right) \bigoplus_{\theta_{j} \in \mathbb{R} \backslash 2 \pi \mathbb{Q}} \theta_{j}\left(\begin{array}{cc}
0 & 1 \\
-1 & 0
\end{array}\right),
$$

where we separated rational and irrational (relative to $2 \pi$ ) values of the NC parameter. To avoid the overcompensations mentioned above we have to assume that irrational $\theta_{j}$ satisfy the Diophantine condition, i.e. that there are two constants $C>0$ and $\beta \geq 0$ such that for all nonzero $q \in \mathbb{Z}$

$$
\inf _{k \in \mathbb{Z}}\left|\theta_{j} q-2 \pi k\right| \geq \frac{C}{|q|^{1+\beta}} .
$$

Generic form of the Diophantine condition which does not rely on a particular block-diagonal form (17) of $\Theta$ can be found in [23]. The Diophantine condition means that $\theta_{j}$ cannot be too well approximated by rational numbers and controls behavior of the exponent in (16). If this condition is violated, the asymptotics of the heat trace are unstable (see Appendix B of [23]), and, therefore, this case is not considered here.

Next we define a trace (which is proportional to the Dixmier trace)

$$
\operatorname{Sp}(L(l) R(r))=\sqrt{g} \sum_{q \in \mathcal{Z}} l_{-q} r_{q},
$$

where the set $\mathcal{Z} \subset \mathbb{Z}^{m}$ consists of all $q$ such that $(2 \pi)^{-1} \Theta q \in \mathbb{Z}^{m}$. Then, one can demonstrate, that

$$
T(\bar{l}, \bar{r})=\operatorname{Sp}(L(\bar{l}) R(\bar{r}))
$$

up to exponentially small terms in $t$. Moreover, in terms of this trace the heat kernel coefficients look precisely as in the commutative case! In particular, all odd-numbered coefficients vanish, and

$$
\begin{aligned}
& a_{0}(l, r, D)=(4 \pi)^{-m / 2} \operatorname{Sp}(L(l) R(r)), \\
& a_{2}(l, r, D)=(4 \pi)^{-m / 2} \operatorname{Sp}(L(l) R(r) E), \\
& a_{4}(l, r, D)=(4 \pi)^{-m / 2} \frac{1}{12} \operatorname{Sp}\left(L(l) R(r)\left(6 E^{2}+2 \nabla^{2} E+\hat{\Omega}^{\mu \nu} \hat{\Omega}_{\mu \nu}\right)\right) .
\end{aligned}
$$

Here $\hat{\Omega}_{\mu \nu}=\nabla_{\mu} \nabla_{\nu}-\nabla_{\nu} \nabla_{\mu}=L\left(\Omega_{\mu \nu}^{L}\right)-R\left(\Omega_{\mu \nu}^{R}\right)$.

Let us stress that all heat kernel coefficients are non-local.

By using the results described above one can calculate the spectral action for a $U(1)$ gauge field on $\mathbb{T}_{\Theta}^{4}$ [23]. Rather unexpectedly, all non-local terms disappear, and one gets the standard action of NC QED. 


\subsection{Finite-temperature manifolds}

To describe finite temperature effects in quantum field theory one often uses the imaginary time formalism where the Euclidean time variable is periodic with the period equal to the inverse temperature. For this reason, one studies $\mathrm{NC}$ quantum field theory on $\mathbb{R}^{3} \times S^{1}$. The most interesting case is, of course, when a compact coordinate on $S^{1}$ does not commute with a non-compact coordinate. The heat kernel expansion for the operator (7) with $\rho=\lambda=0$ was considered in [40] assuming for simplicity that non-compact coordinates on $\mathbb{R}^{3}$ commute between themselves. It was found that planar coefficients still have the same form as above, see (14) and (15). In the mixed sector odd-numbered non-local coefficients appear. Even-numbered mixed coefficients vanish in this case.

\subsection{Isospectral deformation manifolds}

Isospectral deformation $[13,14]$ is a way to define a Moyal-like star product on curved manifolds. Suppose, we have several commuting isometries acting on a manifold. Then one can define a star product which includes derivatives only with respect to the coordinates which correspond to the isometries. One can do some spectral theory on such manifolds as well. In particular, one can analyze the Dixmier traces [22], analyze the mixing between ultra violet and infra red scales by the heat kernel methods [18], and calculate some of the heat kernel coefficients [23]. It is interesting to note, that if the action of the isometries has fixed points, there are specific contributions to the heat kernel expansion coming from these points or submanifolds.

One can define a Moyal-like star product also for a position dependent noncommutativity, but then $\Theta$ must be degenerate [20]. The heat kernel expansion was analyzed on such manifolds [20] by using the methods of covariant perturbation theory [4].

\section{Other NC spaces}

\subsection{Noncommutativity and the standard model of elementary particles}

Noncommutative geometry can provide an explanation of the spectrum of particles in the standard model. In the works devoted to this approach it is assumed that space-time is the product of a four-dimensional manifold by a finite noncommutative space. The heat-kernel part of the calculations of the spectral action is quite standard. Therefore, we do not go into details here. It is worth noting that the coincidence with the observed properties of elementary particles is remarkable. The interested reader may consult the paper [9] and references therein.

\subsection{Field theories with an oscillator potential}

To achieve renormalizability of $\phi^{4}$ theory on $\mathbb{R}_{\Theta}^{4}$ at all orders of perturbation theory one has to modify the action by adding an oscillator potential term [29]

$$
S_{\text {o.p. }}=\frac{\Omega^{2}}{2} \int d^{4} x\left(\tilde{x}^{\mu} \phi\right) \star\left(\tilde{x}_{\mu} \phi\right),
$$

where $\tilde{x}_{\mu}=2(\Theta)_{\mu \nu}^{-1} x^{\nu}$, and $\Omega$ is a parameter. This term leads to an additional potential in $D$ which behaves as $x^{2}$. In the presence of a potential which grows at infinity the heat kernel expansion is modified essentially even in the commutative case. In the NC case, the heat kernel expansion was constructed in $[27,28]$ and used to study an induced gauge field action (in the spirit of the spectral action principle, see Section 2). 


\subsection{Fuzzy spaces}

Roughly speaking, fuzzy spaces are constructed by taking the harmonic expansion on a usual compact space and truncating it at a certain level. This truncated set of the harmonics is not closed under the usual point-wise product, and the most non-trivial part of this construction is to introduce a new associative product on this set which tends to the usual product when the truncation goes to infinity. For example, in the case of fuzzy $S^{2}$ [34] this is done in the following way. Let $\tilde{x}^{a}$ be coordinates on $\mathbb{R}^{3}$ subject to the restriction $\sum_{a=1}^{3}\left(\tilde{x}^{a}\right)^{2}=1$ (so that $\tilde{x}^{a}$ become coordinates on $S^{2}$ ). Any function on $S^{2}$ can be expanded in a sum of homogeneous polynomials of $\tilde{x}^{a}$. By restricting the order of these polynomials to less than some number $n$ one obtains functions on a fuzzy version of $S^{2}$. A suitable multiplication is obtained by identifying $\tilde{x}^{a}$ with certain elements of the algebra of complex $n \times n$ matrices. The restriction to lowest harmonics makes it hard to distinguish points on the two-sphere, so it becomes "fuzzy".

From the point of view of the heat kernel expansion one property of the fuzzy spaces is most important: the spaces of functions are finite dimensional. Consequently, the heat trace $\operatorname{Tr}\left(e^{-t D}\right)$ becomes a finite sum, and in the limit $t \rightarrow 0$ no inverse powers of $t$ may appear. However, Sasakura [37, 38] has demonstrated numerically that the expansion (2) appears for intermediate values of $t$. Correct heat kernel coefficients (3)-(5) were reproduced both for $f=1$ [37] and $f \neq$ const [38] for a number of fuzzy spaces. Analytical understanding of this fact is still missing.

\subsection{Curved Moyal spaces}

The most obvious way to model a curved Moyal space is to replace in (7) the constant metric $g^{\mu \nu}$ by an operator $L\left(g^{\mu \nu}\right)$ with a metric $g^{\mu \nu}(x)$ which depends on NC coordinates. Spectral properties of such operators in a two-dimensional case were analyzed in [46]. Though one can define suitable Dirac and Laplace operators and formulate relations between them (e.g., a generalization of the Lichnerowicz formula), practical calculations of the heat kernel coefficients become very cumbersome. The best one can do is to impose the conformal gauge condition on the metric, $g^{\mu \nu}=e^{-2 \rho} \delta^{\mu \nu}$, and calculate the coefficient $a_{2}$ as power series expansion in the conformal factor $\rho(x)$. There seems to be no obvious relation of this coefficient to geometric invariants. It could be that difficulties with analyzing spectral properties of operators on curved Moyal spaces are related to known problems with formulation of a gravity theory on such spaces (for a review, see [41]).

From the technical point of view, the difficulties with constructing the heat kernel expansion in the case under consideration are caused by deformation of the leading symbol (the part with the highest explicit derivatives) of the Laplacian. Similar problems appear in another version of NC gravity which considers $g^{\mu \nu}$ which as a matrix in some internal space for each pair $(\mu, \nu)$ (see, [2]). Relevant operators become non-minimal (strictly speaking, they are not Laplacians any more). Despite some recent progress [3], actual calculations of the heat trace asymptotics for such operators remain a complicated task.

\section{Application: renormalization and anomalies}

To study one-loop effects in quantum field theory the background field method is frequently used. One decomposes all fields $\phi$ in their background values $\varphi$ and quantum fluctuations $\delta \phi$, $\phi=\varphi+\delta \phi$. Then one expands the classical action retaining the part which is quadratic in $\delta \phi$ only. The result can usually be presented in the form $\int d^{m} x(\delta \phi) D(\delta \phi)$ where the operator $D$ depends on $\varphi$. A typical form of $D$ in $\mathrm{NC}$ theories is given by (7). For example, in $\mathrm{NC} \phi^{4}$ theory $l_{1} \sim r_{1} \sim \varphi \star \varphi$ and $l_{2} \sim r_{2} \sim \varphi$. The one loop effective action is given formally by 
ln $\operatorname{det}(D)$, and, in the framework of the zeta function regularization [16, 30], its' divergent part is proportional to an unsmeared heat kernel coefficient

$$
W_{\mathrm{div}}=-\frac{1}{2 s} a_{m}(D)
$$

where $s$ is a regularization parameter which should be taken 0 at the end of the calculations. The divergences appearing in the limit $s \rightarrow 0$ must be absorbed in redefinitions of the parameters in classical action. To make this possible, $a_{m}(D)$ should repeat the structure of the classical action. (On a side note, let us remark that in the framework of the spectral action principle $a_{m}$, together with other coefficients, defines the classical action, cf. Section 2). In the case of NC $\phi^{4}$ on $\mathbb{R}_{\Theta}^{4}$ planar heat kernel coefficients $a_{4}^{L}(D)$ and $a_{4}^{R}(D)$ satisfy this requirement, and the corresponding mixed coefficient vanishes, $a_{4}^{\operatorname{mix}}(D)=0$. We can conclude that there are no problems with oneloop renormalization of $\mathrm{NC} \phi^{4}$ on the plane (see [47] for details and further references).

On the NC torus $\mathbb{T}_{\Theta}^{4}$ the situation is more subtle. By substituting the functional form of $l_{1,2}$ and $r_{1,2}$ given in the previous paragraph in (18) one obtains (for pure Diophantine $\Theta$ ) nonlocal divergent terms of the form $\int \varphi \cdot \int \varphi \star \varphi \star \varphi$ and $\left(\int \varphi^{2}\right)^{2}$. By imposing anti-periodic conditions on $\varphi$ one can achieve $\int \varphi=0$ and thus kill one type of the divergences. Next, to achieve matching between one-loop divergences and the classical action we add a term $\left(\int \phi^{2}\right)^{2}$ to the classical action. Fortunately, this procedure closes: the new term in the classical action does not generate new types of the one-loop divergences. The renormalizability at this order is restored [23].

As an example of applications of the heat kernel expansion to anomalies and anomalous actions in NC theory let us consider calculations of an induced Chern-Simons action on $\mathbb{T}_{\Theta}^{3}$ [48]. The one-loop effective action for 3-dimensional Dirac fermions is defined by determinant of the Dirac operator

$$
\not D=i \gamma^{\mu}\left(\partial_{\mu}+i L\left(A_{\mu}^{L}\right)+i R\left(A_{\mu}^{R}\right)\right) \text {. }
$$

A peculiar feature of the approach we follow here is the presence of two $U(1)$ gauge fields, $A_{\mu}^{L}$ and $A_{\mu}^{R}$, which are naturally coupled to two conserved currents [48]. It is known [1,36, 15] that the parity-violating part of the effective action (the parity anomaly) reads

$$
W^{\mathrm{pv}}=i \frac{\pi}{2} \eta(0)
$$

where the $\eta$-function is defined as a sum over the eigenvalues $\lambda_{n}$ of the Dirac operator: $\eta(s)=$ $\sum_{\lambda_{n}>0}\left(\lambda_{n}\right)^{-s}-\sum_{\lambda_{n}<0}\left(-\lambda_{n}\right)^{-s}$. By repeating the arguments known from the commutative case [1], one can show that variation of the eta invariant under small variations of the gauge fields is

$$
\delta \eta(0)=-\frac{2}{\sqrt{\pi}} a_{2}\left(\delta \not D, \not D^{2}\right) .
$$

In the zeta regularization, $W^{\mathrm{pv}}=\frac{1}{2} S_{\mathrm{CS}}$, where $S_{\mathrm{CS}}$ is the Chern-Simons action. Now, let us suppose that $\Theta^{12}=-\Theta^{21} \equiv \theta, \Theta^{13}=\Theta^{23}=0$, and that $\theta /(2 \pi)=P / Q$ is rational. Then, by combining the formulae above with known heat kernel coefficients on NC torus (18) one obtains, that the Chern-Simons action in this case consists of three terms $S_{\mathrm{CS}}=S_{\mathrm{CS}}^{L}+S_{\mathrm{CS}}^{R}+S_{\mathrm{CS}}^{\operatorname{mix}}$, where first two terms depend exclusively on $A^{L}$ or $A^{R}$ respectively and coincide with induced ChernSimons actions on the NC plane $[10,26]$. The third, mixed, term reads

$$
S_{\mathrm{CS}}^{\operatorname{mix}}=-\frac{i}{2 \pi} \int d^{3} x \sqrt{g} \epsilon^{\mu \nu \rho}\left[A_{\mu}^{L}\right]_{Q} \partial_{\nu}\left[A_{\rho}^{R}\right]_{Q}
$$


It depends on the parts $\left[A_{\mu}^{L, R}\right]_{Q}$ of the gauge fields which are periodic in $x^{1}$ and $x^{2}$ with the period $(2 \pi) / Q$. Such fields remind us of solid state physics and correspond to a crystal consisting of $Q \times Q$ fundamental domains.

More examples of applications of the heat kernel expansion to renormalization and anomalies in $\mathrm{NC}$ theories, as well as comparison to the results obtained by other methods and further references can be found in $[18,19,20,23,27,39,40,46,47,48]$.

\section{Conclusions and open problems}

As we have already seen, the heat kernel expansion on NC spaces looks much more complicated than in the commutative case. The very structure of the expansion depends crucially on compactness of the manifold and on whether $\Theta^{\mu \nu}$ is degenerate or not. In the compact case, the number theory aspects play an important role. Nevertheless, the case of the Moyal product and of flat noncommutative dimensions is understood quite well, though general analytic formulae are not always available.

At the same time, very little is known about the case when the $\mathrm{NC}$ coordinates are curved (i.e., when the Riemann metric depends on these coordinates). Even a proper generalization of the Laplace operator is still missing. The same is true for a generic position dependent NC parameter $\Theta^{\mu \nu}$ which leads to a star product of the Kontsevich type [33]. The problem seems to be deeper than just a technical one. In these two cases, even classical actions, symmetries and the structure of invariants, quantization rules are still to be determined. Note, that understanding of the heat kernel expansion may in turn help to solve the problems mentioned above (e.g., through the spectral action principle).

\section{Acknowledgements}

This work was supported in part by FAPESP (Brazil).

\section{References}

[1] Alvarez-Gaume L., Della Pietra S., Moore G.W., Anomalies and odd dimensions, Annals Phys. 163 (1985), $288-317$.

[2] Avramidi I.G., Dirac operator in matrix geometry, Int. J. Geom. Methods Mod. Phys. 2 (2005), 227-264, math-ph/0502001.

[3] Avramidi I., Branson T., Heat kernel asymptotics of operators with nonLaplace principal part, Rev. Math. Phys. 13 (2001), 847-890, math-ph/9905001.

[4] Barvinsky A.O., Vilkovisky G.A., Covariant perturbation theory. 2. Second order in the curvature. General algorithms, Nuclear Phys. B 333 (1990), 471-511.

[5] Branson T.P., Gilkey P.B., The asymptotics of the Laplacian on a manifold with boundary, Comm. Partial Differential Equations 15 (1990), 245-272.

[6] Branson T.P., Gilkey P.B., Vassilevich D.V., The asymptotics of the Laplacian on a manifold with boundary. 2, Boll. Union. Mat. Ital. 11B (1997), 39-67, hep-th/9504029.

[7] Branson T.P., Gilkey P.B., Kirsten K., Vassilevich D.V., Heat kernel asymptotics with mixed boundary conditions, Nuclear Phys. B 563 (1999), 603-626, hep-th/9906144.

[8] Chamseddine A.H., Connes A., The spectral action principle, Comm. Math. Phys. 186 (1997), 731-750, hep-th/9606001.

[9] Chamseddine A.H., Connes A., A dress for SM the beggar, arXiv:0706.3690.

[10] Chu C.S., Induced Chern-Simons and WZW action in noncommutative spacetime, Nuclear Phys. B 580 (2000), 352-362, hep-th/0003007.

[11] Connes A., $C^{*}$-algèbres et géométrie différentielle, C.R. Acad. Sci. Paris 290 (1980), 599-604. 
[12] Connes A., Noncommutative geometry, Academic Press, London and San Diego, 1994.

[13] Connes A., Landi G., Noncommutative manifolds, the instanton algebra and isospectral deformations, Comm. Math. Phys. 221 (2001), 141-159, math.QA/0011194.

[14] Connes A., Dubois-Violette M., Noncommutative finite-dimensional manifolds. I. Spherical manifolds and related examples, Comm. Math. Phys. 230 (2002), 539-579, math.QA/0107070.

[15] Deser S., Griguolo L., Seminara D., Gauge invariance, finite temperature and parity anomaly in $D=3$, Phys. Rev. Lett. 79 (1997), 1976-1979, hep-th/9705052.

[16] Dowker J.S., Critchley R., Effective Lagrangian and energy momentum tensor in de Sitter space, Phys. Rev. D 13 (1976), 3224-3232.

[17] Essouabri D., Iochum B., Levy C., Sitarz A., Spectral action on noncommutative torus, arXiv:0704.0564.

[18] Gayral V., Heat-kernel approach to UV/IR mixing on isospectral deformation manifolds, Ann. Henri Poincaré 6 (2005), 991-1023, hep-th/0412233.

[19] Gayral V., Gracia-Bondia J.M., Ruiz F.R., Trouble with space-like noncommutative field theory, Phys. Lett. B 610 (2005), 141-146, hep-th/0412235.

[20] Gayral V., Gracia-Bondia J.M., Ruiz F.R., Position-dependent noncommutative products: classical construction and field theory, Nuclear Phys. B $\mathbf{7 2 7}$ (2005), 513-536, hep-th/0504022.

[21] Gayral V., Iochum B., The spectral action for Moyal plane, J. Math. Phys. 46 (2005), 043503, 17 pages, hep-th/0402147.

[22] Gayral V., Iochum B., Varilly J.C., Dixmier traces on noncompact isospectral deformations, J. Funct. Anal. 237 (2006), 507-539, hep-th/0507206.

[23] Gayral V., Iochum B., Vassilevich D.V., Heat kernel and number theory on NC-torus, Comm. Math. Phys. 273 (2007), 415-443, hep-th/0607078.

[24] Gilkey P.B., Invariance theory, the heat equation, and the Atiyah-Singer index theorem, CRC Press, Boca Raton, FL, 1995.

[25] Gilkey P.B., Asymptotic formulae in spectral geometry, Chapman \& Hall/CRC, Boca Raton, FL, 2004.

[26] Grandi N.E., Silva G.A., Chern-Simons action in noncommutative space, Phys. Lett. B 507 (2001), 345-350, hep-th/0010113.

[27] Grosse H., Wohlgenannt M., Noncommutative QFT and renormalization, J. Phys. Conf. Ser. 53 (2006), 764-792, hep-th/0607208.

[28] Grosse H., Wohlgenannt M., Induced gauge theory on a noncommutative space, hep-th/0703169.

[29] Grosse H., Wulkenhaar R., Renormalisation of $\phi^{4}$ theory on noncommutative $\mathbb{R}^{4}$ in the matrix base, Comm. Math. Phys. 256 (2005), 305-374, hep-th/0401128.

[30] Hawking S.W., Zeta function regularization of path integrals in curved space-time, Comm. Math. Phys. 55 (1977), 133-148.

[31] Kirsten K., The $a_{5}$ heat kernel coefficient on a manifold with boundary, Classical Quantum Gravity 15 (1998), L5-L12, hep-th/9708081.

[32] Kirsten, K., Spectral functions in mathematics and physics, Chapman \& Hall/CRC, Boca Raton, FL, 2001.

[33] Kontsevich M., Deformation quantization of Poisson manifolds, I, Lett. Math. Phys. 66 (2003), 157-216, q-alg/9709040.

[34] Madore J., The fuzzy sphere, Classical Quantum Gravity 9 (1992), 69-88.

[35] Nest R., Vogt E., Werner W., Spectral action and the Connes-Chamsedinne model, Lect. Notes Phys. 596 (2002), 109-132.

[36] Niemi A.J., Topological terms induced by finite temperature and density fluctuations, Phys. Rev. Lett. 57 (1986), 1102-1105.

[37] Sasakura N., Heat kernel coefficients for compact fuzzy spaces, JHEP 2004 (2004), no. 12, 009, 9 pages, hep-th/0411029.

[38] Sasakura, N., Effective local geometric quantities in fuzzy spaces from heat kernel expansions, JHEP 2005 (2005), no. 3, 015, 26 pages, hep-th/0502129.

[39] Strelchenko A., Heat kernel of non-minimal gauge field kinetic operators on Moyal plane, Internat. J. Modern Phys. A 22 (2007), 181-202, hep-th/0608134. 
[40] Strelchenko A.V., Vassilevich D.V., On space-time noncommutative theories at finite temperature, Phys. Rev. D 76 (2007), 065014, 12 pages, arXiv:0705.4294.

[41] Szabo R.J., Symmetry, gravity and noncommutativity, Classical Quantum Gravity 23 (2006), R199-R242, hep-th/0606233.

[42] Vassilevich D.V., Vector fields on a disk with mixed boundary conditions, J. Math. Phys. 36 (1995), 31743182, gr-qc/9404052.

[43] Vassilevich D.V., Heat kernel expansion: user's manual, Phys. Rep. 388 (2003), 279-360, hep-th/0306138.

[44] Vassilevich D.V., Non-commutative heat kernel, Lett. Math. Phys. 67 (2004), 185-194, hep-th/0310144.

[45] Vassilevich D.V., Spectral problems from quantum field theory, Contemp. Math. 366 (2005), 3-22, math-ph/0403052.

[46] Vassilevich D.V., Quantum noncommutative gravity in two dimensions, Nuclear Phys. B 715 (2005), 695712 , hep-th/0406163.

[47] Vassilevich D.V., Heat kernel, effective action and anomalies in noncommutative theories, JHEP 2005 (2005), no. 8, 085, 19 pages, hep-th/0507123.

[48] Vassilevich D.V., Induced Chern-Simons action on noncommutative torus, Modern Phys. Lett. A 22 (2007), 1255-1263, hep-th/0701017. 